\title{
A columnar model explaining long-term memory
}

\author{
Tetsuya Hoshino ${ }^{a}$ Toyohiko Yatagai $^{b}$ and Masahide Itoh $^{a}$ \\ ${ }^{a}$ Institute of Applied Physics, University of Tsukuba, 1-1-1 Tennoudai, Tsukuba, Ibaraki 305-8577, Japan \\ ${ }^{b}$ Center for Optical Research and Education, Utsunomiya University, 7-1-2 Yoto, Utsunomiya, Tochigi
}

321-8585, Japan

e-mail: hoshino@gabor.bk.tsukuba.ac.jp

\begin{abstract}
A hologram provides a useful model for explaining the associative memory of the brain. Recent advances in neuroscience emphasize that single neurons can store complex information and that subtle changes in neurons underlie the process of memorization.
\end{abstract} Experimental results suggest that memory has a localized character. This finding is inconsistent with the characteristics of holographic memory, because this memory has a delocalized, uniform distribution of refractive index in the recorded medium. The recently proposed columnar memory model has a discrete distribution of refractive index. In this study, we first examined the performance of columnar memory and found that it was comparable to holographic memory. Secondly, we showed that this model could explain the above-mentioned experimental results as well as associative memory.

Keywords: holographic memory, columnar memory, brain model, long-term memory, neuron. 


\section{INTRODUCTION}

The number of transistors of the von Neumann-type supercomputer exceeds 10 billion [1].

On the contrary, the number of neurons that make up the human brain is between 50 and 100 billion $[2,3]$, of which 10 billion belong to the cerebral cortex $[4,5]$. This number is too low to explain the brain's high performance capacity if each individual neuron is assumed to correspond to 1 bit, that is, ON/OFF. The human brain is superior to the supercomputer and has wide performance potential [6]. The brain is likely to work on a different scheme from conventional computers. Studies of the human brain may be useful to guide the development of a next-generation computer. Although many neural models of human memory have been presented [7-11], we do not completely understand the mechanism of brain memory yet [12], in part because most research focuses on the local connections between neurons. We believe that a comprehensive theory of the brain's memory mechanisms would be useful and that a model should relate neurons directly to memory in order to explain these mechanisms. If such a model is concrete and valid, we can then apply the model for conducting experiments on the actual brain. Further, the results of these experiments can provide feedback to refine the model.

The holographic memory model is one of the few models that directly and concretely 
combine neurons with contents of memory. Although brain signals are produced by a complex branch of neurons [13], the holographic model suggests that the basic unit of memory is the periodic structure. Actually, the shape of the cat striate cortex (V1) is represented by a sinusoidal wave, and this is important for signal processing in the brain [14]. However, we noticed that the holographic memory model could not explain 2 common experimental results. First, single neurons can store complex information [15], and second, subtle changes in neurons underlie the process of memorization [16]. To address these discrepancies, we present a new model that combines columnar memory [17] and holographic memory. Columnar memory can have various sizes and a complex shape. Furthermore, we estimated the relative performance of the columnar and holographic memory models, because the theoretical limits of memory densities have not yet been compared.

\section{THE PRINCIPLE OF LONG-TERM MEMORY IN THE BRAIN}

The human brain has both short-term memory, lasting several minutes, and long-term memory, lasting decades [18-21]. The signal for memory used in the brain is a pulse train of electrical potentials with an average period in the order of microseconds [22, 23]. These electric potentials can have an interference pattern, as can electric fields [24]. Previous studies have investigated the mechanism responsible for the maintenance of a mammal's 
memory for decades $[19,20]$; these studies have shown that the lifetime of a signal based on electrical potential is not enough to explain the maintenance of long-term memory [19].

Another model to explain memory is the quantum memory model [25]. There is some discussion as to whether the brain works as a quantum computer or not $[26,27]$. Can quantum memory explain long-term memory? The brain has abundant nuclear spin of protons, but the lifetime of this spin is as small as several hundred milliseconds [28], even under a strong magnetic field. Electronic spin is also a candidate mechanism for memory but has a shorter life than nuclear spin $[29,30]$.

As mentioned above, the lifetime of electric signals or the spin state is not enough to account for the maintenance of long-term memory. The change in the shape of a plastic neuron $[16,31]$ has the possibility to last a long time, as it does not require extra energy to maintain its state. This is, therefore, promising as an explanation of long-term memory. The distribution of refractive index in the recording medium can underlie the working of a model of long-term memory.

\section{CHARACTERISTICS OF HOLOGRAPHIC MEMORY}

As a memory model of refractive index, holographic memory is the most popular high-density memory model. It has been attracting attention in the field of recording since it is appropriate for explaining associative [32-34] and delocalized memory; these 
characteristics are similar to those of the human brain $[24,34-36]$. It has a conventional coding method in itself and has a correspondent concrete memory system [32, 37, 38]. It does not need a transistor to perform the calculation, but needs optical elements such as lens and photosensitive polymer $[39,40]$. Hologram information is a Fourier-transformed picture, with its refractive index distribution spreading over a record layer. For example, the associative memory of "goldfish" may be made of many aspects like, face, body, red, etc. All of these are present in the space domain and are Fourier transformed into the frequency domain $[33,34]$ for recording them to the same area. If one of the aspects such as the fish face is projected to the recording area, the other aspects regenerate [34]. This is the function of the associative memory of a hologram.

The distribution of refractive index in a microscopic area is optically Fourier transformed by scattering [41]. This effect is available for the light computing of addition and subtraction $[40,42,43]$. Because holographic memory works by light, it can perform parallel information processing differently from the information processing of a transistor circuit [39].

However, holograms differ from the brain in the following 3 points. Firstly, in the brain, single neurons appear to be related to complex memory [15], but the information of holographic memory is dispersed into the record layer uniformly. Secondly, the distribution of a neuron is discrete [24, 44], but holographic memory is continuous. Moreover, the distribution of electrical potential in a neuron is important for information processing [24]. 
The distribution of the electric potential changes periodically in the column, as the distribution of the electrical field does [24, 45]. The shape of the scatterer (neuron) should affect the electric field largely. If the continuous data of a hologram is represented by discrete neurons, the distances between the neurons should be the same so that Fourier transformation (FT) of the data is easy. Thirdly, the distribution of the refractive index of holographic memory should be changed drastically by memorizing [46], but the change in neurons induced by memorizing is subtle [16]. Let us think of 2 slits as a memorized picture. They are represented holographically as Eq. (1) [47], where " $A$ " is a constant, " $d$ " is the distance between the slits, $\theta$ is the scattering angle in Fig. $1, \lambda$ is the wavelength, and $f(d)$ gives the light intensity distribution.

$$
f(d)=2 A^{2}\{1+\cos [2 \pi d \sin (\theta) / \lambda]\}
$$

When $\sin (\theta)$ changes, the distribution becomes like the hologram of Fig. 2. The subtle change in the distance between the 2 slits alters the hologram a lot.

The above 3 points may not be problematic if the hologram's information is presented by electric signals or distribution of ions, but these memories do not last decades or years.

\section{THE STRUCTURE OF THE HUMAN BRAIN AND ITS MEMORY MODEL}


Despite all the above-mentioned studies, the relationship between memory and the neuron is not clear. The main problem is the ambiguous localization of memory. Hologram is a candidate memory model, but it has the problems described above.

Here, we focus on the structure of the neurons with regards to memory [2].The diameter of a neuron in the brain is several $\mu \mathrm{m}$ [44], which is 100 times thicker than a wire of a transistor. Neurons seem to be unsuitable for storage of high-density memory, but the system of multivalued memory may resolve this problem. For example, using the small change in distance as information, the amount of information in 1 distance might be 10 times more than that in a 1-bit type memory of a neuron.

Human memory has the following 2 structural characteristics.

(1) The sizes and shapes of neurons are categorized into 4 types only [16, 44].

(2) The shape changes induced by memorization are subtle [16].

The neuron itself does not hold the shape of the picture, necessarily. The information may be encoded into different information [48]. As the electric signal in neurons consists of pulse trains [2], the coding can be performed by electric signals. Moreover, the signal from long distance neurons is amplified [16]. This suggests 2 mechanisms; that distances between neurons can provide information and that the information travels long distances. The shape of neurons is complex and columnar. This complex shape can affect the electrical potential within the neuron. It seems unsuitable for an element of optical memory. In order to realize 
optical memory with similar structures and signals to those in the brain, the following may be required.

(1) The distance between columns (neurons) and shape of the columns provides information.

(2) To increase the information capacity, the precision of distance is high.

The problem is the method to measure the distance precisely.

\section{ARRAY OF COLUMNS FOR HIGH-DENSITY MEMORY}

Itoh et al. proposed the model of memory shown in Fig. 1a [17]. This model has the following characteristics.

(1) The shape of the scatterer is columnar.

(2) The information is encoded in the distance between a specific scatterer and the others.

This memory is a multivalued memory, whose information is distance. Because the shape is columnar, and we can consider the change of scattering according to the shape and size of the column, memory can reflect the subtle changes in the column. The electrical field in the column is periodic, and the position of the node affects the scattering character [45]. We think of the specific scatterer as the original point. The distance from the original point to other scatterers encodes information. 
If 1 neuron, which works as the original point, is related to many other neurons, it can store complex data. Actually, single neurons of the human brain have the potential to represent complex information such as one's grandmother's face [49].

This memory is realized if we can measure the distance rapidly and precisely from the scattering pattern. Conventionally, it has been difficult to rigorously calculate the scattering intensity of isolated scatterers other than spheroid scatterers [50, 51]. Rigorous coupled wave analysis (RCWA) is one of the most rigorous calculation algorithms for scattering intensity, but the calculations are generally limited to periodic scatterers [52]. Therefore, Itoh et al. developed a new simple method to apply RCWA to an isolated system [17]. This method enables the calculation of distance by a one-time FT of the distribution of scattering and some correction for the shape of scatterers by RCWA. Moreover, it can utilize the effects of size and shape to extract specific distance information [17]. If the sizes and shapes of columnar scatterers are similar, and the distance between scatters ranges from 1 to several 10 wavelengths, the scattering distribution is approximately calculated by FT of the image, and the abovementioned optical memory is possible. Now, we know that the scattering distribution of the columnar memory can be treated as holographic memory, because holographic memory and a picture are converted into each other by FT.

If the refractive index distribution does not include information about orientation, then information about the arrangement of the original scatterers cannot be extracted by FT and 
analyses of the scattering of recorded information of refractive index distribution. Not only columnar memory, but also holographic memory utilizes FT and obtains information about orientation by using 2 lights with different vectors. The reconstructed image of a hologram has directional information in itself and seems to have the same three-dimensional structure as the original image [53]. In contrast, the columnar memory system measures the distance from a specific scatterer to the others, using interference between scatterers. Complete information regarding the direction is given when the marked scatterer is located on the edge of the scatterers, because a marked scatterer located at the center of the scatterers cannot be used to differentiate between 2 scatterers in opposite directions from each other. The specific scatterer is marked by enhancing the scattering light. It causes the light of the first vector, and the other scatterers cause the light of the second vector.

Figure 1 shows an example of the calculation using this type of memory model. The distance from rectangle 1 to rectangles 2, 3, and 4 is 3, 4, and 5, in this order in Fig. 1a, when wavelength is 1 . The projection of a small image has the effect of FT [41], and the Fourier-transformed image is projected onto the screen. We can obtain the distance by a one-time FT of the distribution on the screen, and the correction can be performed by RCWA. The data has an almost periodic distribution against $\sin (\theta)$, when there are 2 scatterers [17]. Here $\theta$ is the scattering angle, and is defined in Fig. 1a. 
To calculate scattering with directional information, a large left scatterer was added. It has

a strong scattering intensity, because the scattering intensity increases as the scatterer becomes larger [17]. The result of FT is shown in Fig. 1b. We introduced a new parameter, $w_{\text {calc }}$ and set the horizontal axis as $w_{\text {calc }}$ divided by wavelength $\lambda$. The result marked by arrows indicates 3, 4, and 5 in the horizontal axis, and these are the distances between the scatterers shown in Fig. 1a [17]. In this way, the distances from a left scatterer with a large diffraction intensity to the other scatterers are selectively determined.

\section{HOLOGRAPHIC AND COLUMNAR MEMORY}

Holograms records information, into which the original image is already Fourier transformed. For example, a goldfish with its face and body is recorded to the same area as the Fourier-transformed form, as depicted in Fig. 3a. When the picture of the face is projected to the hologram, the body of the goldfish appears, as shown in Fig. 3b. Gabor regenerated the original data in scattering light, using the FT effect of scattering [34]. In contrast, columnar memory records original information itself, as shown in Fig. 4a, and the original data is restored by FT of the distribution of the scattering light. The original point has a stronger scattering intensity than the others. To recall the goldfish, Fig. 4a is projected into the temporal memory, and the temporal memory is projected to the region for recognition again. 
How does columnar memory represent the goldfish and associative memory? In Figs. 3 and 4, we set the face of goldfish, body, and the original point as $\mathrm{A}(x, y), \mathrm{B}(x, y)$, and $\mathrm{C}(x, y)$ in this order in the Cartesian coordinates for the electric field in the plane of the hologram. In holographic memory, when $A$ and $B$ are projected to the plane, the amplitude is $|A+B|^{2}$. If $A$ is projected to it, $\mathrm{B}$ regenerates [34]. When $\mathrm{A}, \mathrm{B}$, and $\mathrm{C}$ are projected to the plane, the amplitude is $|A+B+C|^{2}$. We assume that $|C|^{2}$ is constant $C_{0}$ and $A A *$ is 1 . If $A$ is projected to it, $\mathrm{C}_{0} \mathrm{~A}+\mathrm{B}+\mathrm{C}$ regenerates as Eq. (2) shows.

$$
\mathrm{A}|\mathrm{A}+\mathrm{B}+\mathrm{C}|^{2}=\mathrm{A}|\mathrm{C}|^{2}+\mathrm{A}|\mathrm{A}+\mathrm{B}|^{2}+\mathrm{C}+\mathrm{A}(\mathrm{A}+\mathrm{B}) \mathrm{C}^{*}+\mathrm{AB}^{*} \mathrm{C} .
$$

In this case, if memory regions A and B overlap, they do not separate. Then, in Fig. 4, it is advantageous for the recall of $\mathrm{B}$, not to include $\mathrm{C}$ for projection. We mention the above results concretely in the following. When we project the fish and original point to temporal memory, we get the whole fish, and the original point as associative information, by projecting the face of the fish to the memory. On the contrary, when only the fish is projected, we get only the picture of the body of the fish.

How high is the density of this memory compared with that of the 1-bit memory? Tanaka et al. compared the densities of 1-bit memory and holographic memory theoretically. They observed the multilayer of 1-bit memory by focusing the incident light onto the scatterer in the plane of the specific record layer [54]. They also realized the multilayer of hologram by changing the incidence angle of the reference light discretely [54]. They estimated the 
densities of these three-dimensional optical memories and showed that the densities were not markedly different. Here, we estimated the memory density in 2 dimensions only, because it was difficult to consider the effects of scattering on the other layers, which degraded the signal intensity. We evaluated columnar memory, in addition to holographic memory. Regarding association, the optical system of columnar memory is similar to that of holographic memory. The main difference is that the recorded memory is columnar in shape.

If we represent these 2 memories by the position of neurons, the relative performances are dependent on the precision, resolution, and memory size. We use the bit as an information unit. We use bit density as the information unit per square wavelength. If multivalued memory is superior to two-valued memory, optical memory can have advantages over the two-valued memory of the transistor. We estimated the precision, resolution, and bit density. We compare the performance of single-value and multivalued memory in table 1 . Two-valued memory is 1-bit memory such as Blu-ray, and multivalued memories are holographic memory and columnar memory. Here, we do not assume to increase the multiplicity of memory by an overwritten layer or multilayer structure [55]. We calculated the resolution of 1-bit memory according to the Rayleigh criterion [56]. This resolution is $0.61 \lambda / \mathrm{NA}[56]$, when a lens is used for picking up the scattered light. NA is the numerical aperture, and is at most 1 in the air [57]. We estimated the memory size of 1-bit memory for $\mathrm{NA}=0.76$ 
For columnar memory, we thought of an array of columns as shown in Fig. 5 for memory

content. The distance between the $\left(i^{-} 1\right)$ th column and the $i$-th column is given by

$y_{i}+d_{x}\left(X_{i-1}-x_{i-1}+x_{i}\right)$, using the wavelength as the unit. Here, $x_{0}=0$, and $X_{0}=0 . i=1,2$, $3 \cdots$, and $x_{i}=0,1,2,3 \cdots X_{i} . d_{x}$ is the precision, and $y_{i}$ should be more than, or equal to, the resolution. The precision enhances by 0 filling in FT and it can be $0.01 \lambda$ [17]. The resolution of the columnar memory is $0.8 \lambda$ [17]. The bit number per wavelength is given by Eq. (3).

$$
g\left(X_{i}, y_{i}\right)=\left[\log _{2}\left(X_{i}+1\right)\right] \div\left[y_{i}+d_{x} X_{i}\right]
$$

We set $y_{i}=$ resolution and $X_{i}=X_{1}$ for every $i$. When $X_{1}$ is a parameter, $g\left(X_{1}, y_{1}\right)$ has the maximum value $g\left(X_{\max }, y_{1}\right)$. Using $X_{\max }$, we calculated the bit density, as shown in the table. $X_{\max }$ becomes 31 and $g\left(X_{\max }, y_{1}\right)$ is 4.5 in table 1 . The maximum change of column position becomes $28 \%$ against $\left(y_{1}+d_{\mathrm{x}} X_{\max }\right)$. Here, the information is the distance from the original point to the detail of the picture in Fig. 3.

In contrast, holographic memory is Fourier transformed to give the distance. Before considering the resolution, let us think of the angular distribution of scattering by 2 columns. The hologram and array of columns can be approximately connected by FT and inverse FT [17]. The function is approximated by Eq. (1) [47]. It is the simplest hologram. The sinusoidal convex should be resolved so that the column regenerate by inverse FT. Therefore, we can roughly estimate the resolution of the hologram by the resolution of columnar 
memory. When it is more than $0.8 \lambda[17]$, each convex of the hologram is optically recognized. Let us think of resolution from the viewpoint of producing the hologram. We can make the simplest hologram by using 2 coherent lights with incidence angles $\theta_{1}$ and $\theta_{2}$, as shown in Fig. 2. The resulting period is $\lambda /\left|\sin \left(\theta_{1}\right)-\sin \left(\theta_{2}\right)\right|[53]$. When we make the hologram with period $0.8 \lambda, \theta_{1}$ and $\theta_{2}$ become $-38.7^{\circ}$ and $38.7^{\circ}$, respectively.

When we inversely Fourier transform Eq. (1), 2 data points per period are necessary according to the Whittaker-Shannon sampling theorem[41], and 2 periods are necessary for the FT analysis. If the periods for analysis are smaller than 2, the peak scales out of the graph in Fig. 1b. Therefore, 4 points are necessary. The resolution and required number of points decide the bit density.

As to the bit density in Table 1, holographic memory has a bit density as high as 1-bit memory. This result is not far from that of Tanaka et al. [54]. Columnar memory thus has more than 4 times the density of 1-bit memory.

Finally, we think the maximum change of the peak position of the hologram. Fourier transformed hologram makes the memory in Fig.5. Ignoring the constant term, when hologram is made from two columns, the refractive index distribution is approximately described by Eq. (1). The first peak at $\theta=2 \pi$ changes $28 \%$ against $d / \lambda$ and the second peak at $\theta=4 \pi$ changes $56 \%$. When the distances include $d$ and $5 d$, the change reaches $140 \%$ at $\theta=2 \pi$. 


\section{ASSOCIATIVE MEMORY AND COLUMNAR MEMORY}

Columnar memory is similar to the memory in the brain, because it is discrete and delocalized memory. Moreover, it can make a variety of shapes, and fine control of the scattering pattern may enable the high-level record mode. In this type of memory, the effect of the associative memory-like holography becomes possible by recording the Fourier-transformed image to the temporal memory region [34]. If it is temporal memory, the information can be represented by electric signals or distribution of ions, because the memory does not need a long lifetime. However, here, we use distribution of the refractive index instead of the electric signal as the model of temporary memory in order to make the memory model simple and consistent. For the actual memory system, photosensitive polymer can be used as the recording material of the hologram.

\section{THE BRAIN AND COLUMNAR MEMORY}

We discuss brain memory using a columnar memory model, expecting that we can get good match for the mechanism of the memory. This model requires the columnar scatterer of the original point to be on the edge of the scatterers, so that the positions of the other scatterers are identified. Actually, the hippocampus and peripheral cortex are located at the bottom of the brain [58, 59] in monkeys, and these regions play an important role in physiological memory $[60,61]$. In this model, associative memory can be realized in 2 steps. 
The first step is projecting the information of the goldfish, including the face, body etc. to the same region of the temporal memory. The associative information comes back by the second step of projecting the relative picture onto the temporal memory. In fact, the association process seems to consist of 2 steps of cognition and judgment for the primate memory, and the direction of the electric signal flow is opposite [62]

In a previous study, a monkey was trained to associate 2 different pictures [63]. In this monkey, the neuronal correlates of associative long-term memory were located in the inferior temporal (IT) cortex [63], which is located beside the peripheral cortex. Single neurons in the IT cortex create linkages between representations of 2 different visual stimuli [63]. This may imply that the neuron of "goldfish" itself, or its location, include information on the connection between the specific neurons having information on the shape and idea of the goldfish. Then the IT cortex may be a candidate for the original point in Fig. 4a in monkeys. The medial temporal lobe of humans has a similar function [15], and is located adjacent to the peripheral cortex.

The location of the original point was suggested above. Then, the next interest is the location where the temporal memory for projection resides. Parietal activations are seen frequently in functional neuroimaging studies of episodic memory; on the other hand, parietal lesions do not normally yield severe episodic-memory deficits [61]. This is an unresolved problem. The function of the parietal cortex of humans and monkeys is similar [59]. If the 
place of episodic memory is the parietal cortex, and it works as the temporal associative memory, we can say that the parietal lesions in temporal memory do not affect the episodic memory to a great degree.

\section{CONCLUSIONS}

We have compared holographic memory with columnar optical memory in relation to the brain's long-term memory. Columnar memory is similar to memory formed by an array of neurons in the following 3 ways: the columns are discrete memory, periodical fluctuation of electric fields exists in the columns, and subtle changes to columns can represent information.

The change of position of the column is more subtle for multiple values than that of hologram, considering that hologram is made of trigonometric functions. Moreover, the density of this multivalued memory is more than 4 times higher than that of single-valued memory or holographic memory. Thus, columnar memory is useful as the model of brain memory. We present the combination of columnar memory and holographic memory as a model for memory in the brain to add the function of association to the columnar memory. In this model, columnar memory works as long-term memory, and holographic memory works as temporary memory. 
This model explains why single neurons can encode complex information, as does the grandmother cell. Moreover, it provides a scheme to explain primate associative memory and explains why the memory region may work even if it is damaged.

Thus, we paid attention to the neuron's structure and to the relationship between neurons and the memory, so that we were able to devise the optical memory system suitable for the brain. This memory is simple and concrete and is more similar to brain memory than holographic memory.

\section{Acknowledgements}

The authors gratefully acknowledge Prof. Aoki for discussing on observation of the shape of the neurons. 


\section{REFERENCES}

1. M. Kameyama, "Lithography and itrs," Technical Report 10-06, Institute of Innovation

Research Hitotsubashi University 2010. IIR Working Paper.

2. F. E. Bloom, A. Lazerson, and L. Hofstadter, Brain, mind, and behavior W.H. Freeman, New York, 1985.

3. F. A. Azevedo, L. R. Carvalho, L. T. Grinberg, J. M. Farfel, R. E. Ferretti, R. E. Leite,W. J.

Filho, R. Lent, and S. Herculano-Houzel, "Equal numbers of neuronal and nonneuronal cells make the human brain an isometrically scaled-up primate brain," J. Comp. Neurol. , 2009, vol.

513:, pp. 532-541.

4. G. M. Shepherd, The Synaptic Organization of the Brain Oxford University Press, New York, 2003, 5th ed.

5. P. L. Nunez and R. Srinivasan, Electric Fields of the Brain: The Neurophysics of EEG

Oxford University Press, New York, 2005.

6. H. Moravec, "When will computer hardware match the human brain?" Journal of Evolution and Technology, 1998, vol. 1, pp. 1-10.

7. J. J. Hopfield, "Neural networks and physical systems with emergent collective computational abilities," Proc. Natl Acad. Sci, 1982, vol. 79, pp. 2554-2558.

8. L. F. Abbott and T. B. Kepler, "Model neurons: From Hodgkin-Huxley to Hopfield," Lect. Notes Phys. , 1990, vol. 368, pp. 5-18. 
9. F. Pasemann, “A simple chaotic neuron,” Physica D , 1997, vol. 104, pp. 205-211.

10. P. Heil, H. Neubauer, D. R. F. Irvine, and M. Brown, "Spontaneous activity of auditorynerve fibers: Insights into stochastic processes at ribbon synapses," J. Neurosci. , 1994, vol. 27, pp. 8457-8474.

11. E. T. Rolls and A. Treves, Neural Networks and Brain Function Oxford University Press, Oxford and New York, 1998.

12. J. D. Richter and E. Klann, "Making synaptic plasticity and memory last: mechanisms of translational regulation," Genes \& Dev. , 2009, vol. 23:, pp. 1-11.

13. R. Mizutani, A. Takeuchi, K. Uesugi, S. Takekoshi, R. Y. Osamura, and Y. Suzuki, "Microtomographic analysis of neuronal circuits of human brain," Cereb. Cortex, 2010, vol.20:, pp. 1739-1748.

14. K. S. Sasaki, Y. Tabuchi, and I. Ohzawa, "Complex cells in the cat striate cortex have multiple disparity detectors in the three-dimensional binocular receptive fields," The Journal of Neuroscience , 2010, vol. 30:, pp. 13826-13837.

15. R. Quiroga, L. Reddy, G. Kreiman, and C. Koch, "Invariant visual representation by single neurons in the human brain," Nature , 2005, vol. 435, pp. 1102-1107.

16. Y. Kubota, F. Karube, M. Nomura, A. T. Gulledge, A. Mochizuki, A. Schertel, and Y. Kawaguchi, "Conserved properties of dendritic trees in four cortical interneuron subtypes," Scientific Reports , 2011, vol. 1, pp. 1-13. 
17. T. Hoshino, T. Yatagai, and M. Itoh, "Precise and rapid distance measurements by scatterometry," Opt. Express , 2012, vol. 20, pp. 3954-3966.

18. R. D. Fields, "Making memories stick," Sci. Am. , 2005, vol. 292, pp. 74-81.

19. S. Fusi and L. F. Abbott, "Limits on the memory storage capacity of bounded synapses," Nat. Neurosci., 2007, vol. 10, pp. 485-493.

20. J.-P. Thivierge, F. Dandurand, and D. Cousineau, “A multi-state model of cortical memory," in "Proceedings of International Joint Conference on Neural Networks," IEEE, San Jose, California, USA, 2011, pp. 133-138.

21. M. S. Gazzaniga, The Cognitive NeurosciencesW.W. Norton \& Company, Oxford and New York, 2002, 2nd ed.

22. S. Kuroyanagi and A. Iwata, "Auditory pulse neural network model to extract the interaural time and level difference for sound localization,” IEICE T. Inf. Syst. , 1994, vol. E77-D, pp. 466-474.

23. P. Heil, H. Neubauer, D. R. F. Irvine, and M. Brown, "Spontaneous activity of auditorynerve fibers: Insights into stochastic processes at ribbon synapses," J. Neurosci. , 1994, vol. 27, pp. 8457-8474.

24. P. R. Westlake, "The possibilities of neural holographic processes within the brain," Biological Cybernetics, 1970, vol. 7, pp. 129-153.

25. B. D. E. Artur and Z. Anton, The physics of quantum information: quantum cryptography, 
quantum teleportation, quantum computation Springer Publishing Company, Oxford, 2000.

26. M. Tegmark, "Importance of quantum decoherence in brain processes," Phys. Rev. E, 2000, vol. 61, pp. 4194-4206.

27. C. Koch and K. Hepp, "Quantum mechanics in the brain,” Nature, 2006, vol. 440, pp. $611-612$.

28. J. P. Wansapura, S. K. Holland, R. S. Dunn, and W. S. Ball, "NMR relaxation times in the human brain at 3.0 tesla," J. Magn. Reson.Im. , 1999, vol. 9, pp. 531-538.

29. J. Yin, J. Feix, and J. Hyde, "Mapping of collision frequencies for stearic acid spin labels by saturation-recovery electron paramagnetic resonance," Biophys.J. , 1990, vol. 58, pp. $713-720$.

30. M. Okazaki, K. Kuwata, Y. Miki, S. Shiga, and T. Shiga, "Electron spin relaxation of synthetic melanin and melanin-containing human tissues as studied by electron spin echo and electron spin resonance,” Arch. Biochem. Biophys., 1985, vol. 242, pp. 197-205.

31. C. Leibold and R. Kempter, "Sparseness constrains the prolongation of memory lifetime via synaptic metaplasticity," Cereb.Cortex , 2008, vol. 18, pp. 67-77.

32. D. Gabor, "Holographic model of temporal recall," Nature, 1968, vol. 217, p. 584.

33. D. Gabor, "Improved holographic model of temporal recall," Nature, 1968, vol. 217, pp. $1288-1289$.

34. D. Gabor, “Associative holographic memories,” IBM J. Res. Dev. , 1969, vol. 13, pp. 
$156-159$.

35. K. H. Pribram, Introduction to Fourier optics Lawrence Erlbaum Associates,

Hillsdale,N.J., 1991.

36. K. S. Lashley, Brain mechanisms and intelligence: A quantitative study of injuries to the brain Hafner Pub. Co., New York, 1964.

37. B. Hill, "Some aspects of a large capacity holographic memory," Appl. Opt. , 1972, vol.

11, pp. $182-191$.

38. H. C. Longuet-Higgins, "Holographic model of temporal recall." Nature, 1968, vol. 217,

p. 104

39. D. Psaltis, D. Brady, X.-G. Gu, and S. Lin, "Holography in artificial neural networks,"

Nature, 1990, vol. 343, pp. 325-330.

40. J. Ebersole, "Optical image subtraction,” Optical Engineering , 1975, vol. 14, pp.

$436-447$.

41. J. W. Goodman, Introduction to Fourier optics Roberts and Company Publishers,

Greenwood Village USA, 2005, 3rd ed.

42. D. Gabor, G. W. Stroke, R. Restrick, A. Funkhouser, and D. Brumm, "Optical image

synthesis (complex amplitude addition and subtraction) by holographic Fourier

transformation," Physics Letters , 1965, vol. 18, pp. 116-118.

43. S. H. Lee, S. K. Yao, and A. G. Milnes, "Optical image synthesis (complex amplitude 
addition and subtraction) in real time by a diffraction-grating interferometric method," J. Opt. Soc. Am. , 1970, vol. 60, pp. 1037-1041.

44. H. Zhang, P. L. Hubbard, G. J. Parker, and D. C. Alexander, “Axon diameter mapping in the presence of orientation dispersion with diffusion MRI," NeuroImage, 2011, vol. 56, pp.1301-1315.

45. T. Hoshino, S. Banerjee, M. Itoh, and T. Yatagai, "Design of a wavelength independent grating in the resonance domain," Appl. Opt. , 2007, vol. 46, pp. 7948-7962.

46. M. Born and E. Wolf, Principles of Optics, : Electromagnetic Theory of Propagation, Interference and Diffraction of Light Pergamon Press, Oxford ; New York, 1975, 5th ed. 47. M. Born and E. Wolf, Principles of Optics, : Electromagnetic Theory of Propagation, Interference and Diffraction of Light Cambridge University Press, Cambridge, 1999, $7^{\text {th }}$ ed. 48. J. J. DiCarlo, D. Zoccolan, and N. C. Rust, "How does the brain solve visual object recognition?" Neuron , 2012, vol. 73:, pp. 415-434.

49. C. G. Gross, "Genealogy of the grandmother cell," Neuroscientist, 2002, vol. 8, pp. $512-518$.

50. T. Wriedt, “A review of elastic light scattering theories,” Part. Part. Syst. Char., 1998, vol. 15 , pp. $67-74$.

51. C. F. Bohren and D. R. Huffman, Absorption and scattering of light by small particles Wiley, New York, 1983. 
52. P. Lalanne and E. Silberstein, "Fourier-modal methods applied to waveguide

computational problems," Opt. Lett., 2000, vol. 25, pp. 1092-1094.

53. P. Hariharan, Basics of Holography Cambridge University Press, Cambridge, 2002.

54. T. Tanaka and S. Kawata, "Comparison of recording densities in three-dimensional optical storage systems: multilayered bit recording versus angularly multiplexed holographic recording," J. Opt. Soc. Am. A, 1996, vol. 13, pp. 935-942.

55. M. Gu and X. Li, "The road to bit-by-bit optical data storage," OPN Optics \& Photonics News, 2010, vol. 21, pp. 29-33.

56. E. Hecht, Optics Addison-Wesley, San Francisco, 2002, 4th ed.

57. B. R. Masters, "Ernst Abbe and the foundation of scientific microscopes," Optics \& Photonics News, 2007, vol. 18, pp. 18-23.

58. K. Patterson, P. J. Nestor, and T. T. Rogers, "Where do you know what you know? The representation of semantic knowledge in the human brain," Nature Rev. Neurosci., 2007, vol. 8, pp. 976-988.

59. L. R. Squire, J. T.Wixted, and R. E. Clark, "Recognition memory and the medial temporal lobe: a new perspective," Nature Rev. Neurosci. , 2007, vol. 8, pp. 872-883. 60. C. M. Bird and N. Burgess, "The hippocampus and memory: insights from spatial processing," Nature Rev. Neurosci. , 2008, vol. 9, pp. 182-194.

61. R. Cabeza, E. Ciaramelli, I. R. Olson, and M. Moscovitch, "The parietal cortex and 
episodic memory: an attentional account," Nature Rev. Neurosci. , 2008, vol. 9, pp. 613-625.

62. D. Takeuchi, T. Hirabayashi, K. Tamura, and Y. Miyashita, "Reversal of interlaminar signal between sensory and memory processing in monkey temporal cortex." Science , 2011,vol. 331, pp. 1443-1447.

63. Y. Miyashita, "Cognitive memory: cellular and network machineries and their top-down control." Science , 2004, vol. 306, pp. 435-440. 
Fig. 1. The method used measure the distances [17] (a) The sample array of rectangles. (b) The analyzed results of the angular distribution of scattering. The horizontal axis is the calculated distance. The arrows indicate peaks, which represent the distance from rectangle 1 to rectangles 2,3 , and 4 .

Fig. 2. Schematic diagram depicting the arrangement of 2 incident lights and the hologram.

Fig. 3. The associative memory of a hologram (a) Hologram made from scattering light from the face and body of goldfish. (b) The body is the response associated with the stimulus of the face.

Fig. 4. The columnar memory model for explaining the brain's memory mechanism (a) Content of long-term memory: goldfish and original point. (b) Visual stimulus of the fish face. (c) Hologram of the fish for the temporal memory. (d) Response to the stimulus

Fig. 5. The arrangement of columnar memory. The rectangle is the column. The vertical line indicates the possible position of a column. The distance between the neighboring lines is $d_{x}$. 
Table 1. Comparison of the densities of memory types

\begin{tabular}{lccccc}
\hline $\begin{array}{l}\text { Memory } \\
\text { unit }\end{array}$ & $\begin{array}{c}\text { Information } \\
\text { type }\end{array}$ & $\begin{array}{c}\text { Resolution } \\
(\lambda)\end{array}$ & $\begin{array}{c}\text { Precision } \\
(\lambda)\end{array}$ & $\begin{array}{c}\text { Number of } \\
\text { points }(1 / \mathrm{bit})\end{array}$ & $\begin{array}{c}\text { Bit density } \\
\left(1 / \lambda^{2}\right)\end{array}$ \\
\hline 1 bit & ON/OFF & 0.8 & 0.01 & 1 & 1.6 \\
\hline $\begin{array}{l}\text { Multiple } \\
\text { bit }\end{array}$ & Columns & 0.8 & 0.01 & 0.22 & 7.0 \\
\cline { 2 - 6 } & Hologram & 0.8 & 0.01 & 0.89 & 1.8 \\
\hline
\end{tabular}


(a)

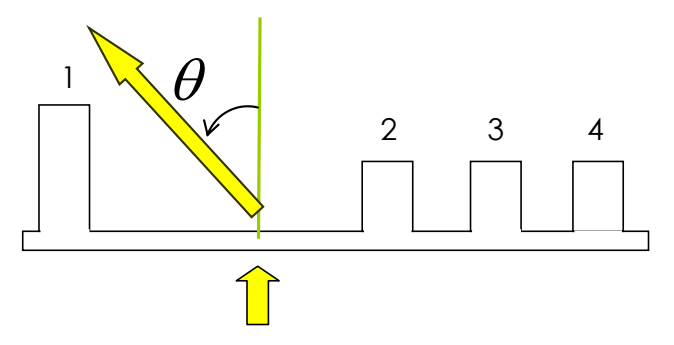

(b)

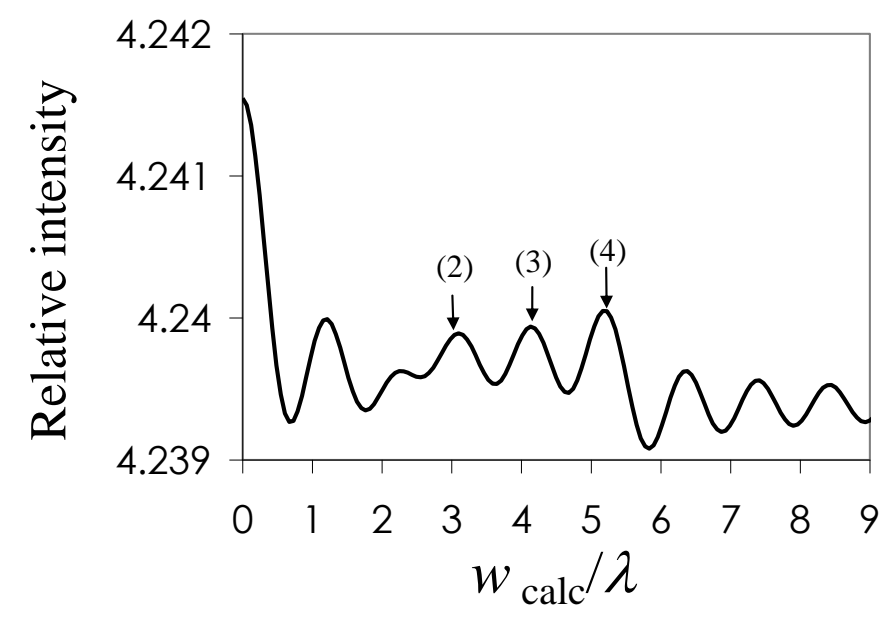

Fig. 1. The method used measure the distances [17] (a) The sample array of rectangles. (b) The analyzed results of the angular distribution of scattering. The horizontal axis is the calculated distance. The arrows indicate peaks, which represent the distance from rectangle 1 to rectangles 2,3 , and 4 . 


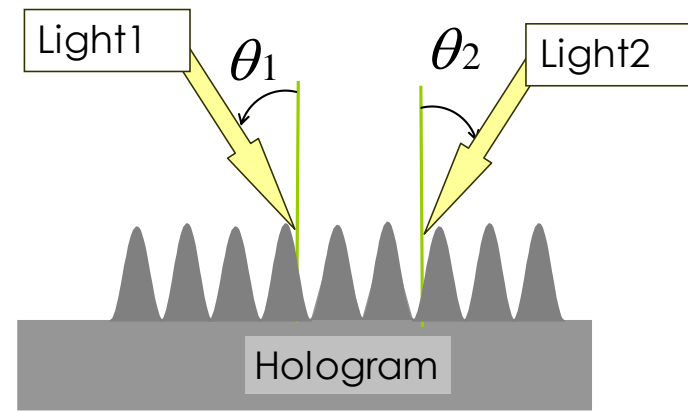

Fig. 2. Schematic diagram depicting the arrangement of 2 incident lights and the hologram. 
(a)

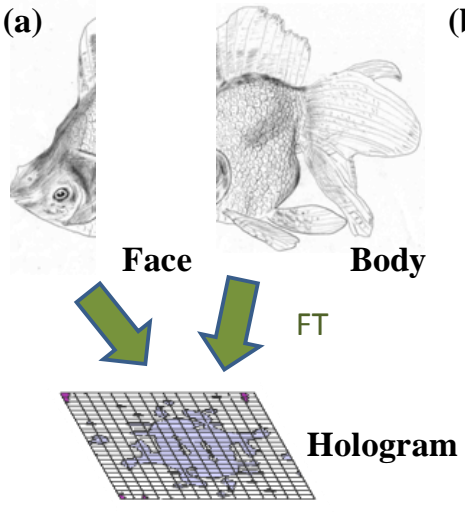

(b)

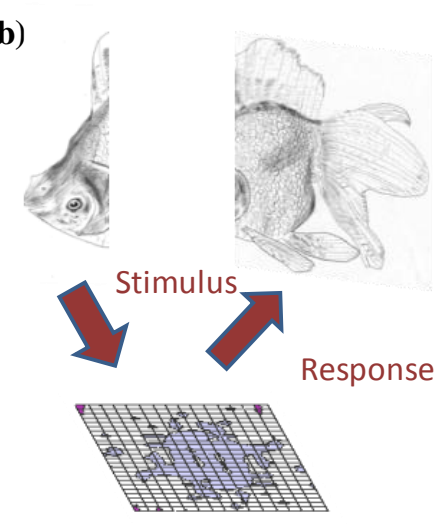

Fig. 3. The associative memory of a hologram (a) Hologram made from scattering light from the face and body of goldfish. (b) The body is the response associated with the stimulus of the face.

(a)

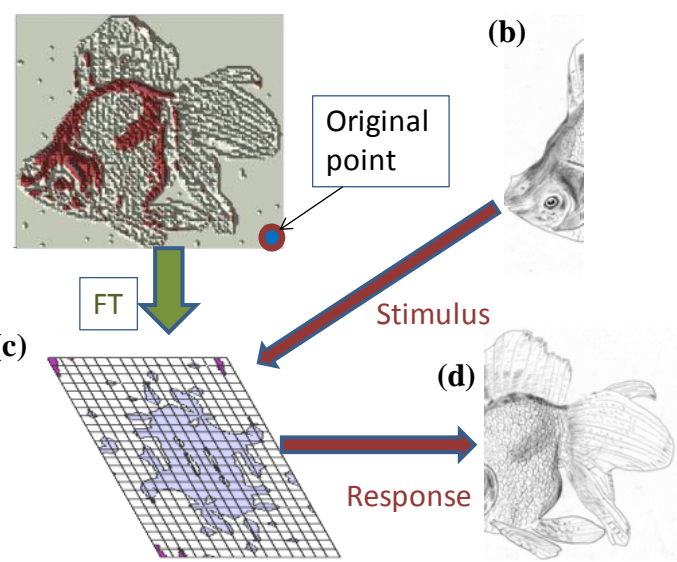

Fig. 4. The columnar memory model for explaining the brain's memory mechanism

(a) Content of long-term memory: goldfish and original point. (b) Visual stimulus of the fish face. (c) Hologram of the fish for the temporal memory. (d) Response to the stimulus. 


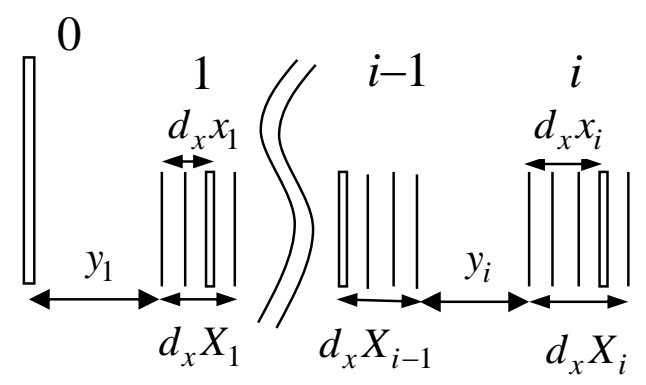

Fig. 5. The arrangement of columnar memory. The rectangle is the column. The vertical line indicates the possible position of a column. The distance between the neighboring lines is $d_{x}$. 